\title{
Epidemiología e impacto de las infecciones nosocomiales
}

\author{
P.M. Olaechea ${ }^{a, *}$, J. Insausti ${ }^{b}$, A. Blanco ${ }^{c}$ y P. Luque ${ }^{d}$

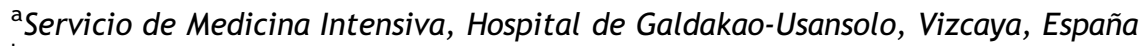 \\ ${ }^{\mathrm{b}}$ Servicio de Medicina Intensiva, Hospital de Navarra, Pamplona, España \\ 'Servicio de Medicina Intensiva, Hospital Universitario Central de Asturias, Oviedo, España \\ ${ }^{\mathrm{d} S}$ Servicio de Medicina Intensiva, Hospital Clinico Universitario Lozano Blesa, Zaragoza, España
}

Recibido el 10 de octubre de 2009; aceptado el 22 de noviembre de 2009

Disponible en Internet el 21 de enero de 2010

\section{PALABRAS CLAVE \\ Infección nosocomial; Paciente crítico; \\ Vigilancia epidemiológica; Impacto de la infección nosocomial}

\section{KEYWORDS}

Nosocomial infection; Critical patient;

Surveillance; Impact of nosocomial infection

\begin{abstract}
Resumen
Las infecciones nosocomiales (IN) son uno de los problemas más importantes que ocurren en las unidades de cuidados intensivos, por lo que es necesario conocer la epidemiología y el impacto que estas infecciones tienen en el paciente crítico. Se toman como base los datos del Estudio Nacional de Vigilancia de Infección Nosocomial en Unidades de Cuidados Intensivos y se hace una descripción de las tasas y de la etiología de las principales IN, como son la neumonía asociada a ventilación mecánica, la infección urinaria asociada a sondaje uretral y la bacteriemia primaria y secundaria. Se hace una revisión de la literatura médica con respecto a las consecuencias de las diferentes IN y se hace especial hincapié en las infecciones causadas por microorganismos multirresitentes.

(c) 2009 Elsevier España, S.L. y SEMICYUC. Todos los derechos reservados.
\end{abstract}

\section{Epidemiology and impact of nosocomial infections}

\section{Abstract}

Nosocomial infections are one of the most important problems occurring in Intensive Care Units. For this reason, the epidemiology and impact of these infections on critical patients must be known. Based on the data from the ENVIN-UCI study, the rates and etiology of the main nosocomial infections, such as ventilator-associated pneumonia, urinary tract infection and primary and secondary bloodstream infection, have been described. A review of the literature regarding the impact of different nosocomial infections on critically ill patients, particularly those caused by multidrug-resistant bacteria, was also performed.

(c) 2009 Elsevier España, S.L. and SEMICYUC. All rights reserved.

\footnotetext{
*Autor para correspondencia.

Correo electrónico: pedromaria.olaecheaastigarrag@osakidetza.net (P.M. Olaechea).
} 


\section{Introducción}

Las infecciones, aunque han abandonado los primeros puestos que ostentaban en el pasado, continúan siendo una importante causa de mortalidad en el mundo. Actualmente, son la segunda causa de muerte tras las enfermedades cardiovasculares ${ }^{1-3}$. Durante el siglo xx la mortalidad por enfermedad infecciosa disminuyó de forma drástica con el consiguiente incremento en la expectativa de vida. Estos cambios se debieron principalmente a la aparición de los antibióticos y a los avances en técnicas diagnósticas y terapéuticas medicoquirúrgicas.

A las infecciones comunitarias se agregan las infecciones nosocomiales (IN) que, en general, afectan a los pacientes más graves. La IN en su definición tradicional es aquella que aparece durante el ingreso hospitalario, pero en la actualidad se extiende también a la que se relaciona con los cuidados sanitarios en un sentido amplio. Estas infecciones son unas veces motivo de ingreso en las unidades de cuidados intensivos ( $\mathrm{UCl})$ y otras, consecuencia de la estancia en éstas.

El mayor problema añadido a estas enfermedades es la aparición de resistencia a los antibióticos de uso común. Esta resistencia es debida principalmente a la abusiva, y en ocasiones inadecuada, utilización de los antibióticos. La presencia de microorganismos multirresistentes (MMR) tiene importantes repercusiones para los pacientes y el sistema sanitario (costes, brotes epidémicos y morbimortalidad). La progresiva disminución del tiempo de hospitalización con una mejora en la externalización de los cuidados sanitarios a la comunidad contribuye a que con mayor frecuencia encontremos MMR en el medio extrahospitalario con patrones de resistencia similares a los nosocomiales.

\section{Infección asociada a los cuidados sanitarios}

Las camas hospitalarias han disminuido como consecuencia del aumento de plazas en otras formas de atención sanitaria. Actualmente, la atención hospitalaria está relacionada con los cuidados ambulatorios, la hospitalización a domicilio, los centros sociosanitarios y de larga estancia e incluso con las instituciones geriátricas. En todos estos ámbitos asistenciales se genera un tipo de infección que se parece más a la nosocomial que a la comunitaria. Esto ha llevado a cambiar el clásico concepto de IN por el más actual de infección asociada a los cuidados sanitarios, que engloba ambos tipos de infecciones. Existe un importante desconocimiento sobre el verdadero impacto de estas infecciones al no disponer de tanta información como la que tenemos referida a los hospitales. Por todo esto, se entiende que no sea suficiente el mantener un estricto control de la IN y la multirresistencia sólo en el medio hospitalario, debe tenerse una visión más global con una coordinación de los esfuerzos en los diferentes ámbitos ${ }^{4}$.

\section{Programas de control de la infección nosocomial}

En el mundo se han desarrollado programas de seguimiento y control de la IN. Uno de los primeros se desarrolló en Estados Unidos mediante los Centers for Disease Control and
Prevention llamado proyecto Study on the Efficacy of Nosocomial Infection Control y buscaba un mejor control de la IN. En una muestra de hospitales americanos ${ }^{5}$, el establecimiento de la vigilancia y del control exhaustivo entre 1970 y 1976 se asoció a una importante reducción en las tasas de IN. Posteriormente, se desarrolló en este país el sistema National Nosocomial Infection Surveillance ${ }^{6}$, que es un sistema voluntario y confidencial sobre el que se realizan informes mensuales de más de 300 hospitales y cuyos datos contribuyen de manera muy importante a detectar cambios en los patrones de incidencia, resistencia a antibióticos, focos, pronóstico y factores de riesgo para la infección.

En Europa se han desarrollado varios sistemas paralelos, cuya información se centralizó durante un tiempo en el proyecto europeo $\mathrm{HELICS}^{7}$. De estos proyectos tienen más relevancia el Krankenhaus Infektions Surveillance System (KISS) de Alemania ${ }^{8}$, el Preventie van Ziekenhuisinfecties door Surveillance (PREZIES) ${ }^{9}$ de Holanda o el Reseau Alerte Investigation Surveillance des Infections (RAISIN) ${ }^{10}$ de Francia. En los últimos años se han iniciado proyectos multinacionales en varios países sudamericanos como el Internacional Nosocomial Infection Control Consortium $(\text { INICC })^{11}$.

En España se han desarrollado principalmente 2 sistemas de vigilancia de IN: el Estudio de la Prevalencia de la IN en España (EPINE) es un estudio de prevalencia que se realiza anualmente desde 1990 y fue el primer sistema de vigilancia desarrollado en el país. Su objetivo es determinar las tendencias en las tasas de las IN en los hospitales españoles ${ }^{12}$.

El Estudio Nacional de Vigilancia de Infección Nosocomial en Servicios de Medicina Intensiva (ENVIN-UCI) desarrollado por el Grupo de Trabajo de Enfermedades Infecciosas de la Sociedad Española de Medicina Intensiva, Crítica y Unidades Coronarias desde 1994 recoge datos de las principales IN asociadas a dispositivos y llegan a participar en la actualidad más de 100 unidades anualmente ${ }^{13}$. Es un sistema específicamente dirigido a la $\mathrm{UCl}$ y en el que son objeto de seguimiento únicamente aquellas infecciones relacionadas directamente con factores de riesgo conocidos o que se asocian a mayor morbimortalidad en pacientes críticos, como son la neumonía relacionada con la ventilación mecánica (NAVM), la infección urinaria relacionada con sondaje uretral (IU-SU), la bacteriemia primaria y relacionada con catéteres vasculares y la bacteriemia secundaria. Los datos provenientes de este estudio se han convertido durante estos años en referencia obligada para evaluar los esfuerzos de mejora que se vienen realizando en esta área y son accesibles en la página web http://hws.vhebron.net/ envin-helics/. La aportación de datos al proyecto europeo HELICS hizo que el ENVIN-UCI se denominara también ENVIN-HELICS.

\section{Indicadores de calidad}

La Sociedad Española de Medicina Intensiva y Unidades Coronarias ha elaborado 100 indicadores que permiten valorar la calidad asistencial que se realiza en cada unidad $^{14}$. Los indicadores de calidad referidos a las enfermedades infecciosas son los siguientes: 
- Bacteriemia relacionada con catéter venoso central (CVC): 4 episodios por 1.000 días de CVC.

- Infección urinaria relacionada con sondaje: 6 episodios por 1.000 días de sonda urinaria.

- NAVM: 18 episodios por 1.000 días de ventilación mecánica.

- Tratamiento antibiótico empírico inadecuado en la IN: $10 \%$.

- Infecciones por Staphylococcus aureus resistente a meticilina (SARM): 0,4 episodios de infección por 1.000 días de estancia.

- Indicación de aislamientos: $100 \%$.

- Tratamiento precoz en la sepsis grave/choque séptico: $95 \%$.

- Administración de corticoides en el choque séptico: $95 \%$.

De los mencionados, los 5 primeros pueden obtenerse mediante el ENVIN-HELICS. Controlar estos indicadores antes y después de las medidas de prevención propuestas nos dará una idea de la utilidad de éstas ${ }^{15}$.

\section{Evolución de las tasas de infección nosocomial}

La evolución en la densidad de incidencia anual de NAVM en el ENVIN-UCI se muestra en la figura 1. Hasta el momento esta tasa no ha disminuido por debajo de 14,5 neumonías por 1.000 días de ventilación mecánica, mínimo alcanzado en el período de estudio correspondiente al año 2007. La evolución de la densidad de incidencia de IU-SU se muestra en la figura 2. Se observa una cierta estabilidad con tasas, en los últimos años, ligeramente inferiores a 5 infecciones por 1.000 días de sondaje uretral. Finalmente, en la figura 3 se refleja la evolución de la densidad de incidencia de la bacteriemia primaria y relacionada con catéteres vasculares conjuntamente. Esta tasa alcanzó un máximo de 8,3 episodios de bacteriemia por 1.000 días de catéter venoso central en 1998. A partir del año 2005 se ha observado una disminución progresiva de esta tasa.

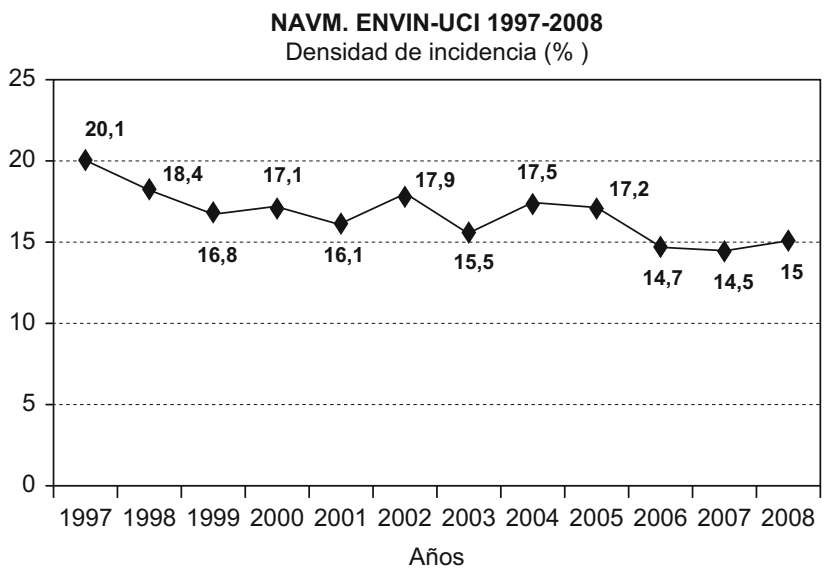

Figura 1 Estudio Nacional de Vigilancia de Infección Nosocomial-Unidad de Cuidados Intensivos. Evolución de la tasa de neumonía asociada a ventilación mecánica (NAVM) en el período 1997-2008.

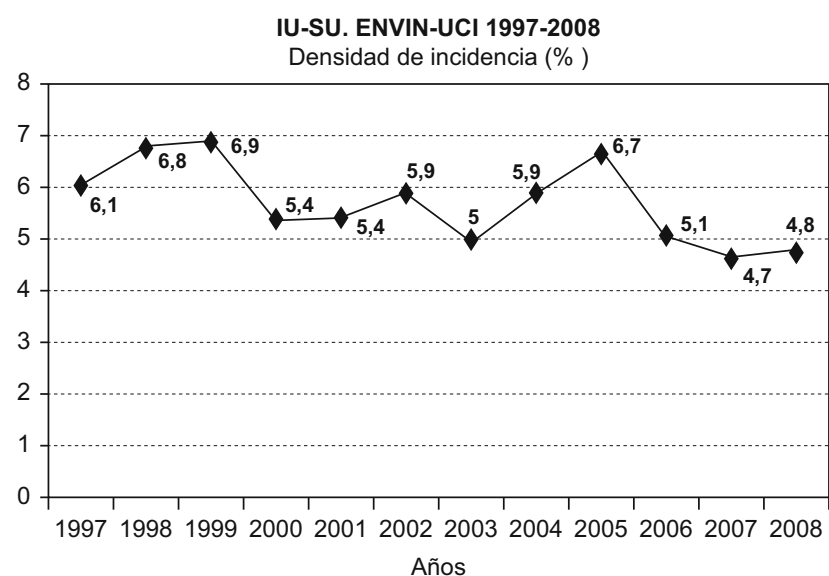

Figura 2 Estudio Nacional de Vigilancia de Infección Nosocomial-Unidad de Cuidados Intensivos. Evolución de la tasa de infección urinaria asociada a sondaje uretral (IU-SU) en el período 1997-2008.

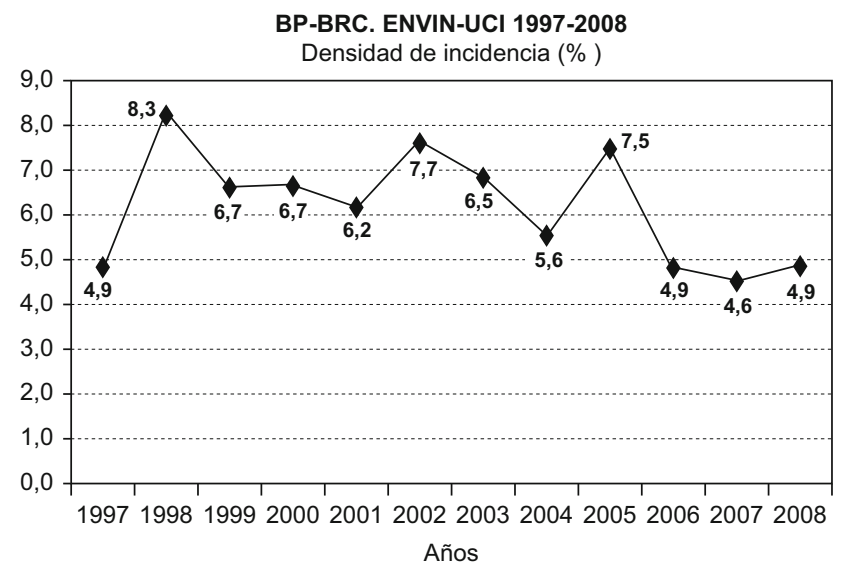

Figura 3 Estudio Nacional de Vigilancia de Infección Nosocomial-Unidad de Cuidados Intensivos. Evolución de la tasa de bacteriemia primaria y relacionada con catéter (BP-BRC) en el período 1997-2008.

\section{Etiología de la infección nosocomial}

Un aspecto esencial de la epidemiología de las IN es el conocimiento de la etiología de estas infecciones y las modificaciones que pueden ocurrir en el tiempo en cada $\mathrm{UCI}$. En el último informe del ENVIN-HELICS referido al año 2008 se analiza la evolución de estas infecciones en 13.824 pacientes $^{16}$. En este último año, con datos de 1.879 infecciones asociadas a dispositivos, el $54,9 \%$ de éstas estaban causadas por bacilos gramnegativos, los grampositivos constituyen el $32,4 \%$ y las infecciones originadas por hongos constituyen el $12,2 \%$ (tabla 1 ). Al considerar los patógenos de forma individual, en el conjunto de los últimos 5 años, el primer lugar lo ocupa Pseudomonas aeruginosa $(13,1 \%)$ seguido de Escherichia coli $(11,3 \%)$, S. aureus $(7,2 \%)$, Staphylococcus epidermidis $(7,1 \%) \mathrm{y}$, en quinto lugar, Candida albicans $(6,0 \%)$ (tabla 2$)$. 
Tabla 1 Grupos de microorganismos en las diferentes localizaciones (Estudio Nacional de Vigilancia de Infección NosocomialUnidad de Cuidados Intensivos en el período 2008)

\begin{tabular}{lccccc}
\hline & Total, n (\%) & NAVM, n (\%) & IU-SU, n (\%) & BP-BRC, n (\%) & BS, n (\%) \\
\hline BGN & $991(54,9)$ & $620(67,2)$ & $248(56,9)$ & $123(27,6)$ & $94(57,3)$ \\
Grampositivos & $585(32,4)$ & $208(22,5)$ & $85(19,5)$ & $292(65,5)$ & $53(32,3)$ \\
Hongos & $221(12,2)$ & $91(9,9)$ & $103(23,6)$ & $27(6,1)$ & $15(9,6)$ \\
Otros & $8(0,4)$ & $4(0,4)$ & $0(0,0)$ & $4(0,9)$ & $2(1,2)$ \\
\hline
\end{tabular}

BGN: bacilos gramnegativos; BP/BRC: bacteriemia primaria y relacionada con catéter; BS: bacteriemia secundaria; IU-SU: infección urinaria asociada a sondaje uretral; NAVM: neumonía asociada a ventilación mecánica.

Tabla 2 Microorganismos aislados en las principales infecciones nosocomiales intraunidad de cuidados intensivos (excluyendo bacteriemias secundarias) (Estudio Nacional de Vigilancia de Infección Nosocomial-Unidad de Cuidados Intensivos en el período 2008)*

\begin{tabular}{|c|c|c|c|c|c|c|}
\hline Año & $\begin{array}{l}2004 \\
n=1.045\end{array}$ & $\begin{array}{l}2005 \\
n=1.585\end{array}$ & $\begin{array}{l}2006 \\
n=1.544\end{array}$ & $\begin{array}{l}2007 \\
n=1.625\end{array}$ & $\begin{array}{l}2008 \\
n=1.805\end{array}$ & $\begin{array}{l}\text { Total } \\
n=7.604\end{array}$ \\
\hline Pseudomonas aeruginosa & $138(13,2)$ & $198(12,5)$ & $190(12,3)$ & $217(13,4)$ & $254(14,1)$ & $997(13,1)$ \\
\hline Escherichia coli & $113(10,8)$ & $184(11,6)$ & $182(11,8)$ & $178(10,9)$ & $201(11,1)$ & $858(11,3)$ \\
\hline Staphylococcus aureus & $88(8,4)$ & $117(7,4)$ & $97(6,3)$ & $122(7,5)$ & $120(6,6)$ & $544(7,2)$ \\
\hline Staphylococcus epidermidis & $83(7,9)$ & $132(8,3)$ & $103(6,7)$ & $101(6,2)$ & $121(6,7)$ & $540(7,1)$ \\
\hline Candida albicans & $60(5,7)$ & $96(6,1)$ & $91(5,9)$ & $87(5,3)$ & $119(6,6)$ & $453(6,0)$ \\
\hline Acinetobacter baumannii & $54(5,2)$ & $96(6,1)$ & $129(8,3)$ & $145(8,9)$ & $100(5,5)$ & $447(5,9)$ \\
\hline Enterococcus faecalis & $52(5,0)$ & $113(7,1)$ & $83(5,4)$ & $78(4,8)$ & $91(5,0)$ & $390(5,1)$ \\
\hline Staphylococcus coagulasa negativa & $65(6,2)$ & $60(3,8)$ & $70(4,5)$ & $81(5,0)$ & $91(5,0)$ & $367(4,8)$ \\
\hline Klebsiella pneumoniae & $35(3,3)$ & $68(4,3)$ & $61(3,8)$ & $80(4,9)$ & $74(4,1)$ & $318(4,2)$ \\
\hline
\end{tabular}

*Número de microorganismos aislados cada año y porcentaje de aislamientos.

Tabla 3 Microorganismos predominantes en la neumonía asociada a ventilación mecánica (Estudio Nacional de Vigilancia de Infección Nosocomial-Unidad de Cuidados Intensivos en el período 2004-2008)*

\begin{tabular}{lllllll}
\hline Año & $\begin{array}{l}2004 \\
\mathrm{n}=506\end{array}$ & $\begin{array}{l}2005 \\
\mathrm{n}=\mathbf{7 4 3}\end{array}$ & $\begin{array}{l}2006 \\
\mathrm{n}=\mathbf{7 2 8}\end{array}$ & $\begin{array}{l}2007 \\
\mathrm{n}=832\end{array}$ & $\begin{array}{l}\text { 2008 } \\
\mathrm{n}=\mathbf{9 2 3}\end{array}$ & $\begin{array}{l}\text { Total } \\
\mathrm{n}=\mathbf{3 . 7 3 2}\end{array}$ \\
\hline $\begin{array}{lllll}\text { Pseudomonas aeruginosa } \\
\text { SASM }\end{array}$ & $92(18,2)$ & $133(17,9)$ & $123(16,9)$ & $146(17,6)$ & $172(18,6)$ & $666(17,8)$ \\
Acinetobacter baumanii & $74(14,6)$ & $92(12,4)$ & $86(11,8)$ & $104(12,5)$ & $104(11,4)$ & $460(12,3)$ \\
Escherichia coli & $38(7,5)$ & $65(8,7)$ & $93(12,8)$ & $98(11,8)$ & $83(9,0)$ & $377(10,1)$ \\
Haemophilus influenzae & $43(8,5)$ & $51(6,9)$ & $49(6,7)$ & $60(7,2)$ & $70(7,6)$ & $273(7,32)$ \\
SARM & $35(6,9)$ & $58(7,8)$ & $43(5,9)$ & $43(5,1)$ & $58(6,3)$ & $237(6,35)$ \\
Klebsiella pneumoniae & $25(4,9)$ & $50(6,7)$ & $54(7,4)$ & $34(4,1)$ & $33(3,6)$ & $196(5,25)$ \\
& $20(3,9)$ & $41(5,5)$ & $30(4,1)$ & $53(6,4)$ & $41(4,4)$ & $185(5,0)$ \\
\hline
\end{tabular}

SARM: Staphylococcus aureus resistente a meticilina; SASM: Staphylococcus aureus sensible a meticilina.

*Número de microorganismos aislados cada año y porcentaje de aislamientos.

En lo que se refiere a la etiología de la NAVM (tabla 3), infección que representa el $45,0 \%$ del conjunto de las IN estudiadas, y al ver la evolución de los últimos 5 años, $P$. aeruginosa $(17,8 \%)$ ocupa la primera posición, seguida por $S$. aureus (12,3\%) y Acinetobacter baumanii $(10,1 \%)$. Existen importantes variaciones etiológicas, pero dependen de que la neumonía sea de aparición precoz ( $\leq 7$ días) o tardía ( $>7$ días). Así, mientras en las precoces domina $S$. aureus sensible a meticilina (SASM), en las tardías lo hace $P$. aeruginosa. Las IU-SU representan el 23,3\% del total de las infecciones investigadas en la UCl. En éstas, $E$. coli es con diferencia el primer agente etiológico con un 28,3\% de los casos, seguido a distancia por C. albicans $(13,2 \%)$ y P. aeruginosa (12,8\%) (tabla 4). En las bacteriemias primarias y relacionadas asociadas a catéteres (el 23,2\% del total de las infecciones), el agente causal más frecuente 
Tabla 4 Microorganismos predominantes en la infección urinaria asociada a sondaje uretral (Estudio Nacional de Vigilancia de Infección Nosocomial-Unidad de Cuidados Intensivos en el período 2004-2008)*

\begin{tabular}{lllllll}
\hline Año & $\begin{array}{l}2004 \\
\mathrm{n}=\mathbf{2 4 3}\end{array}$ & $\begin{array}{l}2005 \\
\mathrm{n}=\mathbf{4 2 8}\end{array}$ & $\begin{array}{l}2006 \\
\mathrm{n}=398\end{array}$ & $\begin{array}{l}2007 \\
\mathrm{n}=\mathbf{2 8 9}\end{array}$ & $\begin{array}{l}2008 \\
\mathrm{n}=\mathbf{4 3 6}\end{array}$ & $\begin{array}{l}\text { Total } \\
\mathrm{n}=\mathbf{1 . 7 9 4}\end{array}$ \\
\hline Escherichia coli & $61(25,1)$ & $112(26,2)$ & $115(28,9)$ & $106(26,2)$ & $113(25,9)$ & $507(28,3)$ \\
Candida albicans & $32(13,4)$ & $56(13,1)$ & $49(12,3)$ & $48(12,3)$ & $51(11,7)$ & $236(13,2)$ \\
Pseudomonas aeruginosa & $38(15,6)$ & $47(11,0)$ & $40(10,1)$ & $45(11,6)$ & $60(13,8)$ & $230(12,8)$ \\
Enterococcus faecalis & $24(9,9)$ & $65(15,2)$ & $43(10,8)$ & $38(9,8)$ & $46(10,5)$ & $216(12,0)$ \\
Candida spp. & $25(10,3)$ & $24(5,6)$ & $31(7,8)$ & $42(10,8)$ & $42(9,6)$ & $164(9,1)$ \\
Klebsiella pneumoniae & $9(3,7)$ & $17(4,0)$ & $18(4,5)$ & $12(3,1)$ & $21(4,8)$ & $77(4,3)$ \\
Proteus mirabilis & $7(2,9)$ & $9(2,1)$ & $10(2,5)$ & $12(3,1)$ & $24(5,5)$ & $62(3,5)$ \\
\hline
\end{tabular}

*Número de microorganismos aislados cada año y porcentaje de aislamientos.

Tabla 5 Microorganismos predominantes en las bacteriemias primarias y relacionadas con catéter venoso central (Estudio Nacional de Vigilancia de Infección Nosocomial-Unidad de Cuidados Intensivos en el período 2004-2008)*

\begin{tabular}{|c|c|c|c|c|c|c|}
\hline Año & $\begin{array}{l}2004 \\
n=296\end{array}$ & $\begin{array}{l}2005 \\
n=407\end{array}$ & $\begin{array}{l}2006 \\
n=414\end{array}$ & $\begin{array}{l}2007 \\
n=404\end{array}$ & $\begin{array}{l}2008 \\
n=446\end{array}$ & $\begin{array}{l}\text { Total } \\
n=1.967\end{array}$ \\
\hline Staphylococcus epidermidis & $75(25,3)$ & $109(26,8)$ & $94(22,7)$ & $92(22,7)$ & $103(23,1)$ & $473(24,1)$ \\
\hline Staphylococcus coagulasa negativa & $59(19,9)$ & $55(13,5)$ & $66(15,9)$ & $71(17,6)$ & $85(19,1)$ & $336(17,1)$ \\
\hline Enterococcus spp. & $25(8,4)$ & $53(13,0)$ & $52(12,6)$ & $38(9,4)$ & $39(8,7)$ & $207(10,5)$ \\
\hline Candida spp. & $17(5,7)$ & $20(4,9)$ & $24(5,8)$ & $22(5,5)$ & $27(6,0)$ & $110(5,6)$ \\
\hline Pseudomonas aeruginosa & $14(4,7)$ & $19(4,7)$ & $29(7,0)$ & $26(6,4)$ & $21(4,7)$ & $109(5,5)$ \\
\hline Otros Staphylococcus & $20(6,7)$ & $18(4,4)$ & $17(4,1)$ & $17(4,2)$ & $31(7,0)$ & $103(5,2)$ \\
\hline Acinetobacter baumanii & $12(4,1)$ & $16(3,9)$ & $26(6,3)$ & $28(6,9)$ & $11(2,5)$ & $93(4,7)$ \\
\hline Escherichia coli & $9(3,0)$ & $20(4,9)$ & $17(4,1)$ & $16(3,9)$ & $18(4,0)$ & $80(4,1)$ \\
\hline Staphylococcus aureus MS & $12(4,1)$ & $20(4,9)$ & $10(2,4)$ & $15(3,7)$ & $11(2,5)$ & $68(3,5)$ \\
\hline Enterobacter cloacae & $5(1,7)$ & $12(3,9)$ & $16(3,9)$ & $12(3,0)$ & $14(3,1)$ & $59(3,0$ \\
\hline Staphylococcus aureus MR & $5(1,7)$ & $18(4,4)$ & $14(3,4)$ & $5(1,2)$ & $7(1,6)$ & $49(2,5)$ \\
\hline
\end{tabular}

MR: resistente a meticilina; MS: sensible a meticilina.

*Número de microorganismos aislados cada año y porcentaje de aislamientos.

es S. epidermidis $(24,1 \%)$, seguido por Staphylococcus coagulasa negativa $(17,1 \%)$ y Enterococcus faecalis $(10,5 \%)$ (tabla 5$)$.

\section{Multirresistencia antibiótica}

La resistencia antibiótica bacteriana fue un proceso continuo que se inició con la resistencia a la penicilina de $S$. aureus. La introducción de las penicilinas resistentes a las penicilinasas en la década de 1960 llevó al desarrollo de resistencia a meticilina y la aparición de SARM. Es el ejemplo más evidente, pero no el único. La evolución global de la multirresistencia, tomados los datos del ENVIN del período 2004-2008, se expone en la tabla 6. Se observa una disminución de las cepas de SARM por segundo año consecutivo y una estabilización de la resistencia a cefalosporina y ciprofloxacino en $E$. coli.

Reviste una especial importancia en nuestro entorno la falta de sensibilidad a meticilina de $S$. aureus. La evolución en la tasa global de SARM en el ENVIN-UCI del período 1994-2008 se muestra en la figura 4, con una tendencia creciente que parece haberse frenado en los 2 últimos años. En Estados Unidos es un verdadero problema de salud pública agravado por la aparición de SARM de origen comunitario. Su aparición se ha seguido de una rápida expansión que afecta a la población más joven y sin factores de riesgo para la adquisición de SARM de origen nosocomial ${ }^{3,17}$. Este patógeno produce infecciones de la piel y de las partes blandas, aunque también pueden provocar neumonías comunitarias graves. Este problema ya ha iniciado su presencia, aunque de momento es escasa en España ${ }^{18}$.

Al considerar la evolución de los distintos marcadores, es de señalar la elevada proporción de resistencia de $A$. baumanii a imipenem, pero también la creciente resistencia de $P$. aeruginosa a antibióticos habitualmente considerados como específicos para este patógeno, sobre todo en lo que respecta a ciprofloxacino (tabla 6).

Como ya se ha mencionado, otro aspecto importante en el campo de la multirresistencia lo constituye la aparición de enterococos (Enterococcus faecium y $E$. faecalis) resistentes a vancomicina, lo que ha llegado a convertirse, en algunos países, en un problema de primer orden ${ }^{2}$. En nuestro país, 
Tabla 6 Evolución de los marcadores de multirresistencia en el período 2004-2008*

\begin{tabular}{|c|c|c|c|c|c|}
\hline Año & 2004 & 2005 & 2006 & 2007 & 2008 \\
\hline SARM & 27,7 & 37,1 & 42,2 & 24,4 & 25,0 \\
\hline Staphylococcus aureus $\mathrm{R}$ a vancomicina & 0 & 0,6 & 0 & 0 & 0 \\
\hline Staphylococcus epidermidis $\mathrm{R}$ a meticilina & 88,1 & 85,2 & 83,6 & 80,9 & 84,1 \\
\hline S. epidermidis $\mathrm{R}$ a vancomicina & 0 & 0 & 0 & 0,7 & 1,9 \\
\hline Escherichia coli $\mathrm{R}$ a ciprofloxacino & 31,5 & 32,1 & 34,4 & 34,4 & 32,4 \\
\hline E. coli $\mathrm{R}$ a cefotaxima & 14,5 & 10,0 & 13,1 & 16,8 & 13,2 \\
\hline Acinetobacter spp. $\mathrm{R}$ a imipenem & 49,1 & 58,3 & 54,6 & 76,4 & 66,3 \\
\hline Pseudomonas aeruginosa $\mathrm{R}$ a amikacina & 7,6 & 11,4 & 13,0 & 12,9 & 17,7 \\
\hline P. aeruginosa $\mathrm{R}$ a ceftazidima & 26,2 & 29,0 & 27,9 & 27,2 & 26,3 \\
\hline P. aeruginosa $\mathrm{R}$ a ciprofloxacino & 31,5 & 30,2 & 33,1 & 35,2 & 38,0 \\
\hline$P$. aeruginosa $\mathrm{R}$ a imipenem & 31,2 & 28,6 & 36,3 & 32,0 & 34,6 \\
\hline P. aeruginosa $\mathrm{R}$ a piperacilina-tazobactam & 24,1 & 22,4 & 18,7 & 18,9 & 14,5 \\
\hline Enterococcus spp. $\mathrm{R}$ a vancomicina & 0 & 1,0 & 0 & 0 & 0 \\
\hline
\end{tabular}

R: resistente; SARM: Staphylococcus aureus resistente a meticilina.

*Porcentaje de cepas resistentes respecto al total del mismo microorganismo identificado en las infecciones controladas.

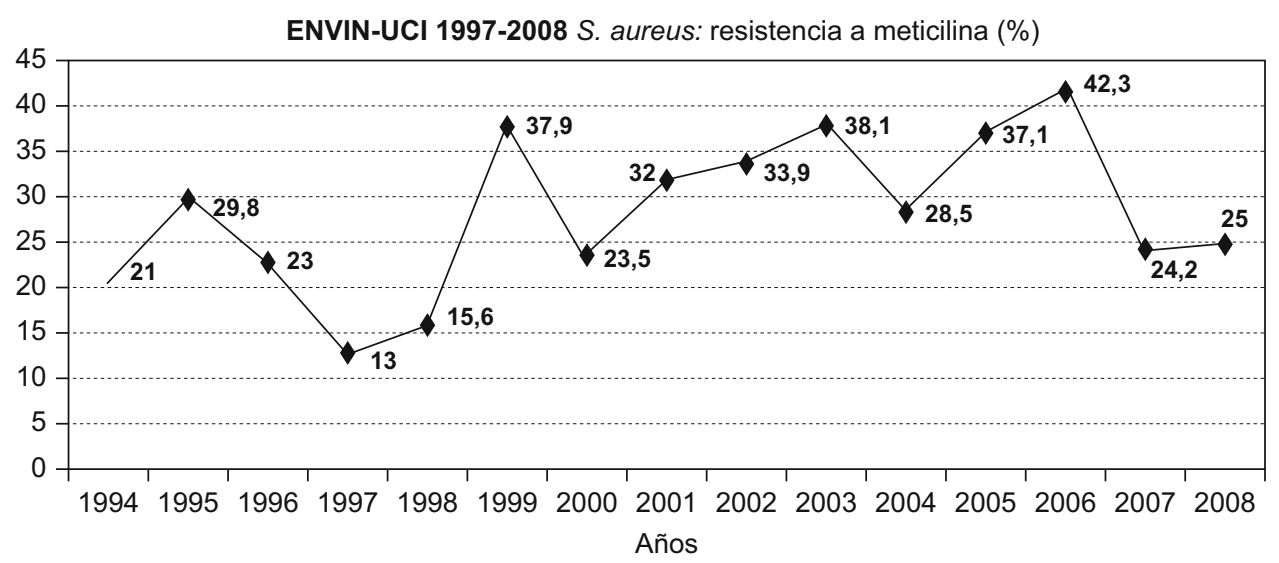

Figura 4 Estudio Nacional de Vigilancia de Infección Nosocomial-Unidad de Cuidados Intensivos. Evolución de Staphylococcus aureus resistente a meticilina/Staphylococcus aureus (\%) en el período 1994-2008.

aunque hace tiempo que se han descrito y se ha referenciado algún brote epidémico, los porcentajes de resistencia son actualmente inferiores al $5 \%{ }^{19,20}$.

El constante incremento de la multirresistencia debe impulsar la búsqueda de nuevas opciones terapéuticas de mayor efectividad y seguridad, al tiempo que se necesita un estrecho seguimiento del perfil de sensibilidad-resistencia de los agentes etiológicos frente a los antimicrobianos que se van incorporando.

\section{Impacto de la infección nosocomial}

La medición del impacto que tiene una IN fue durante muchos años un tema de debate y en el que quedan varios aspectos aún no resueltos. Hay que empezar por preguntarse si realmente es posible medir este efecto, ya que si bien, entendemos fácilmente a lo que nos referimos al hablar de mortalidad (aunque puedan quedar muchas dudas sobre cuándo medirla), el término morbilidad es mucho más difícil de precisar, pero en el que se incluye un conjunto de factores, en buena parte subjetivos, como son el peligro de muerte, el malestar, el dolor o el sufrimiento, la discapacidad temporal o permanente y la disminución de la calidad de vida en el futuro. Aunque no se hace habitualmente, parte de estas consecuencias asociadas a la IN podría medirse mediante cuestionarios validados, incluyendo los aspectos éticos relacionados con las IN y su control $^{21,22}$.

Podría calcularse la morbilidad mediante la medición del consumo de recursos, algo más fácilmente cuantificable, aunque no se haría más que medir un aspecto parcial del impacto global, ya que se mediría solamente el impacto directo (o coste directo), mientras que se olvidan otros aspectos igualmente importantes (impactos indirectos) como son lo relacionado con la prevención de las infecciones (costes de prevención) o los gastos y pérdidas de ingresos futuros para el paciente que tiene una IN. Además, el impacto de la IN debe medirse en diferentes niveles como son en el propio paciente, en el hospital, en la aseguradora (sistema de salud que financia el cuidado del paciente) y la sociedad $^{23,24}$. 
Tabla 7 Factores relacionados con el impacto de la infección nosocomial en el paciente crítico

Edad del paciente

Comorbilidades (diabetes, hepatopatía, insuficiencia renal, EPOC, etc.)

Expectativas de vida

Enfermedad de base

Gravedad del paciente al ingreso en la UCI y en el momento de adquirir la infección

Localización de la infección

Etiología del patógeno causante de la infección

Tiempo de estancia previa a la aparición de la IN

Resistencia antibiótica de los microorganismos causales

Adecuación del tratamiento antibiótico (respecto a la sensibilidad y a la adecuación de la dosis, momento, etc.)

Adecuación de otros tratamientos (retirada de catéter, intervenciones quirúrgicas, tratamiento de ventilación mecánica, etc.)

Número y tipo de otras IN que pudieran adquirirse con posterioridad

Protocolos de alta de la UCl de cada hospital

Protocolos de limitación del esfuerzo terapéutico de cada hospital

EPOC: enfermedad pulmonar obstructiva crónica; IN: infección nosocomial; UCI: unidad de cuidados intensivos.

Medir las consecuencias de las IN es complejo, sobre todo en pacientes ingresados en la UCI. Responder a la pregunta si el paciente fallece (o tiene una estancia más prolongada) con la infección o por la IN requiere valorar un conjunto de variables de confusión que influyen en distinto grado según la infección que se trate (tabla 7) y que pueden agruparse en factores vinculados con el paciente, relacionados con la infección y su agente etiológico, y los conectados con el tratamiento recibido y la evolución posterior, en el que se incluyen protocolos de actuación propios de cada hospital en relación con el alta y con la limitación del esfuerzo terapéutico. Esta complejidad ha propiciado una serie de estudios sobre el proceso mismo de la medición del impacto de la $I^{25,26}$. El problema fundamental es la interrelación entre estos factores, como, por ejemplo, que los MMR aparecen con más frecuencia en pacientes más graves, que han recibido más tratamientos antibióticos y que tienen una estancia hospitalaria más prolongada $y$, por todo esto, tienen de base un peor pronóstico.

\section{Repercusión en el paciente}

\section{Mortalidad}

Existe un consenso en la literatura médica que apunta que la presencia de MMR y la inadecuación del tratamiento antibiótico son los factores más importantes relacionados directamente con el incremento de la mortalidad ${ }^{27-32}$. Pero separar el tratamiento empírico inadecuado de la presencia de MMR es imposible y constituye una de las variables de confusión más complicadas de abordar ${ }^{32,33}$. Además, la propia definición del tratamiento inadecuado es compleja, ya que no solamente se refiere al empleo de antibióticos cuya sensibilidad in vitro es inadecuada, sino también cuando se emplean por vía inadecuada, con retraso, en dosis incorrectas para el paciente o incluso el empleo de antibióticos con mala difusión al foco de infección o que presentan interacciones con otros medicamentos que se suministre al paciente o que no se empleen en combinación cuando esté indicado ${ }^{34}$. Aún con todo, también es posible encontrar trabajos que no han hallado asociación entre tratamiento antibiótico inadecuado y mortalidad ${ }^{35,36}$.

Hace 15 años Fagon et $\mathrm{al}^{37}$ escribieron una revisión sobre el riesgo de fallecer como consecuencia de las IN y afirmaban: “Un análisis preciso indica que: 1) el efecto es altamente probable para la neumonía, dudoso en la bacteriemia e incierto para la infección urinaria; 2) el riesgo se incrementa con la duración de la estancia en la $\mathrm{UCl}$; 3) la etiología bacteriana modifica el riesgo, y 4) el efecto es mayor en pacientes menos graves porque la gravedad de la enfermedad basal es el factor más importante". Esta afirmación sigue siendo válida en la mayoría de los puntos, aunque debe matizarse el primero de éstos a la luz de los conocimientos actuales.

Hay datos discrepantes sobre la influencia de la NAVM en la mortalidad ${ }^{38-47}$ que indican en algunos casos que existe una relación directa entre la infección y la mortalidad y, en otros, no. El motivo de la discrepancia estriba en la selección de la población, pero puede afirmarse que existe una relación directa en 3 circunstancias: 1) la inadecuación del tratamiento antibiótico empírico inicial ${ }^{44-48}$; 2) la etiología de la NAVM ${ }^{42,49}$, y 3) el momento de aparición de la NAVM, ya que tiene más influencia en la mortalidad en las infecciones tardías, pero no en las precoces ${ }^{41,44,47}$. Fuera de estas situaciones, probablemente la NAVM no tiene impacto en la evolución de los pacientes.

Con respecto a las bacteriemias, también hay datos discordantes, ya que se encuentran estudios en los que se describe un incremento de la mortalidad asociada (entre el 10 y el $35 \%$ ) a la bacteriemia nosocomial ${ }^{38,39,50-52}$ y otros que no, cuando se ajusta al resto de las variables de confusión ${ }^{53,54}$. Los factores que influyen en un mayor o en un menor impacto en la mortalidad son la etiología de la bacteriemia, el origen, sea primaria, asociada a catéter o a otros focos y la menor gravedad de los pacientes (más impacto en aquéllos con menor gravedad $)^{55}$. En datos extraídos del ENVIN-HELICS la mortalidad atribuida a la bacteriemia primaria y relacionada con catéter es de un 9,4\%, aunque con un impacto mayor de las bacteriemias primarias y menor de las secundarias a catéter ${ }^{56}$.

Se ha establecido mejor que la IU-SU aparecida en pacientes críticos no tiene impacto en la mortalidad de estos pacientes ${ }^{57,58}$. 
Al centrarnos en la influencia sobre la mortalidad de diferentes MMR, probablemente los más estudiados hayan sido el SARM y su comparación con el SASM. Un metaanálisis de Cosgrove et $\mathrm{al}^{59}$ publicado en el año 2003 demostraba que la probabilidad de fallecimiento de los pacientes con bacteriemia por SARM era casi el doble a la de los pacientes con bacteriemia por SASM. Sin embargo, los mismos autores en una serie de pacientes propia ${ }^{60}$ y publicada un par de años después no encuentran este incremento de mortalidad. Diferentes formas de medir la influencia de la resistencia a meticilina han derivado en diferentes resultados ${ }^{61-64}$. Probablemente los factores más importantes son la adecuación del tratamiento antibiótico ${ }^{65-67}$ o la localización de la infección ${ }^{68}$ como condicionantes de mayor mortalidad en pacientes con infecciones por SARM que por SASM.

También se ha estudiado la influencia de $P$. aeruginosa, especialmente en casos de multirresistencia, en la mortalidad, e igualmente se han encontrado algunos datos contradictorios. Por un lado, la resistencia a imipenem en pacientes con neumonía por $P$. aeruginosa parece ser un condicionante de incremento de mortalidad $^{69,70}$, pero no si la resistencia es a piperacilina-tazobactam ${ }^{71,72}$. Una vez más, la adecuación del tratamiento antibiótico ${ }^{73}$ o la aparición de resistencias durante el tratamiento ${ }^{74}$ son los factores más importantes relacionados con el impacto. A similares conclusiones puede llegarse cuando se refieren a la mortalidad de los pacientes con bacteriemia por $A$. baumanii ${ }^{75,76}$ o cuando existe resistencia a imipenem $^{77-79}$. Sin embargo, otros estudios no han demostrado relación directa entre la infección por este patógeno y la mortalidad ${ }^{80-82}$.

Parece evidente que un retraso en el tratamiento adecuado de infecciones por enterobacterias productoras de betalactamasa de espectro extendido se asocia a un incremento de la mortalidad ${ }^{83-86}$, sobre todo cuando se consideran infecciones de distinta localización a la urinaria ${ }^{84,87,88}$, si bien la falta de estudios controlados limita el valor de la conclusión mayoritaria ${ }^{89}$.

\section{Morbilidad}

Si en el caso de la mortalidad pueden existir dudas en cuanto al impacto real de la infección, en el caso de la cuantificación de la morbilidad no queda ninguna. La presencia de IN siempre se asocia a un incremento de la estancia y de los costes. En general, consideradas globalmente, la IN se asocia a un incremento de la estancia hospitalaria que oscila entre los 5 y los 15 días $^{16,29,30,90-92}$ (de mediana), pero evidentemente varía de acuerdo con la localización de la infección ${ }^{40}$ (probablemente más en NAVM y bacteriemia secundaria y menos en infección urinaria o bacteriemia por catéter), la adecuación del tratamiento antibiótico empírico ${ }^{29}$ y el patógeno causante.

Otro aspecto que también debe tenerse en cuenta son los efectos que puede tener sobre los pacientes la necesidad de aislamiento y el deterioro que supone para la calidad de vida del paciente mientras permanece ingresado y aislado. Se ha cuantificado cómo, en los aislamientos de contacto, los trabajadores sanitarios acuden a la cabecera del paciente la mitad de las veces que a los pacientes no aislados, lo que puede retardar una evolución adecuada y el alta hospitalaria ${ }^{93,94}$. Pero quizá no sea esto lo más importante, sino la situación de desvalimiento que muchas veces tienen los pacientes aislados.

\section{Impacto de la infección nosocomial en otras áreas}

Aparte del impacto directo que cualquier IN tiene sobre el paciente, también hay que tener en cuenta aquellos costes que afectan al paciente por permanecer más días ingresados, su falta de productividad debida al retraso en la incorporación a la vida laboral, los costes de desplazamiento de su familia y cuidadores, etc.

Aunque la forma de medir el impacto económico de una IN varía de unas situaciones sanitarias a otras, en nuestro medio ${ }^{95}$ la medición de la prolongación de estancia puede ser un parámetro adecuado, ya que, en general, refleja los gastos de personal (alrededor de un 70\%), de los medicamentos administrados (10-15\%) o de las pruebas diagnósticas necesarias para el diagnóstico de la infección, pero también debe tenerse en cuenta los gastos que la prevención de las IN lleva consigo, tanto en el mantenimiento de los equipos de vigilancia como en los programas educacionales destinados al personal, programas de intervención en la política antibiótica ${ }^{96}$, empleando el tiempo tanto de quien da como de quien recibe este tipo de formación.

Los pacientes infectados por MMR representan un coste económico por paciente entre los 5.000 y los 25.000 euros, cantidad que está muy por encima de las infecciones causadas por microorganismos sensibles ${ }^{97}$. Estimaciones más recientes proporcionan cifras igualmente variables, éstas son más bajas cuando se relacionan con la estancia hospitalaria previa (alrededor de 3.500 euros de coste incremental) ${ }^{98}$, o son más altas y pueden ascender a más de 8.000 euros cuando se estima la prolongación de la estancia en la $\mathrm{UCl}^{99}$, o cifras que superan los 50.000 euros cuando se miden las consecuencias económicas de las infecciones por $M_{M R}{ }^{78,100}$

La multirresistencia a antibióticos afecta al hospital en 4 aspectos: a) primero, por la necesidad de que cada hospital disponga de equipos y programas de vigilancia de las IN, y se buscan específicamente aquellos patógenos con mayor multirresistencia y con posibilidad de transmisión entre pacientes $^{101}$; b) segundo, porque hace que todo el hospital diseñe e implante un programa de política antibiótica, ya que el mal uso de antibióticos es una de las causas de aparición y persistencia de MMR; c) tercero, porque obliga a mantener aislados a los pacientes con infecciones 0 colonizaciones por MMR y, por tanto, implica una mayor disponibilidad de camas, modificaciones arquitectónicas, consumo de recursos materiales e incremento del personal para atender a estos pacientes, con todo lo que esto implica desde el punto de vista económico, y d) cuarto, porque por la necesidad de aislamiento y riesgo de transmisión son pacientes que requieren trasladarse a espacios específicos (habitaciones de aislamiento dentro del hospital o residencias fuera del ámbito hospitalario), habitualmente con poca disponibilidad y con rechazo por la posibilidad de contagio a otros pacientes, lo que redunda en mantener estos 
pacientes en áreas como las $\mathrm{UCl}$ de forma más prolongada que lo estrictamente necesario.

\section{Conflicto de intereses}

Los autores declaran no tener ningún conflicto de intereses.

\section{Bibliografía}

1. Fauci AS, Touchette NA, Folkers GK. Emerging infectious diseases: A 10-year perspective from the National Institute of Allergy and Infectious Diseases. Emerg Infect Dis. 2005;11: 519-525.

2. Biedenbach DJ, Moet GJ, Jones RN. Occurrence and antimicrobial resistance pattern comparisons among bloodstream infection isolates from the SENTRY Antimicrobial Surveillance Program (1997-2002). Diagn Microbiol Infect Dis. 2004;50: 59-69.

3. Klevens RM, Morrison MA, Nadle J, Petit S, Gershman K, Ray S, et al. Invasive methicillin-resistant Staphylococcus aureus infections in the United States. JAMA. 2007;298:1763-71.

4. Barcenilla F, Jover A, Castellana D, López R. Control de la infección nosocomial. Una visión más allá de cuidados intensivos. En: Net A, Quintana E, editores. Infecciones en Medicina Intensiva. Barcelona: Ars Medica; 2007. p. 19-28.

5. Haley RW, Culver DH, White JW, Morgan WM, Emori TG. The nationwide nosocomial infection rate. A new need for vital statistics. Am J Epidemiol. 1985;121:159-67.

6. National Nosocomial Infections Surveillance (NNIS) System Report, data summary from January 1992 through June 2004, issued October 2004. Am J Infect Control. 2004;32:470-85.

7. Suetens C, Morales I, Savey A, Palomar M, Hiesmayr M, Lepape $A$, et al. European surveillance of ICU-acquired infections (HELICS-ICU): Methods and main results. J Hosp Infect. 2007;65:171-3.

8. Gastmeier P, Sohr D, Schwab F, Behnke M, Zuschneid I, Brandt $C$, et al. Ten years of KISS: The most important requirements for success. J Hosp Infect. 2008;70:11-6.

9. Mannien J, van den HS, Muilwijk J, van den Broek PJ, van Benthem B, Wille JC. Trends in the incidence of surgical site infection in the Netherlands. Infect Control Hosp Epidemiol. 2008;29:1132-8.

10. Astagneau P, L'Heriteau F, Daniel F, Parneix P, Venier AG, Malavaud $S$, et al. Reducing surgical site infection incidence through a network: Results from the French ISO-RAISIN surveillance system. J Hosp Infect. 2009;72:127-34.

11. Rosenthal VD, Maki DG, Graves N. The International Nosocomial Infection Control Consortium (INICC): Goals and objectives, description of surveillance methods, and operational activities. Am J Infect Control. 2008;36:1-12.

12. Rossello-Urgell J, Vaque-Rafart J, Villate-Navarro JI, SánchezPaya J, Martínez-Gómez X, Arribas-Llorente JL, et al. Exposure to extrinsic risk factors in prevalence surveys of hospital-acquired infections: A methodological approach. J Hosp Infect. 2006;62:366-71.

13. Álvarez-Lerma F, Palomar M, Olaechea P, Otal JJ, Insausti J, Cerda E. Estudio Nacional de Vigilancia de Infección Nosocomial en Unidades de Cuidados Intensivos. Informe evolutivo de los años 2003-2005. Med Intensiva. 2007;31:6-17.

14. Martin MC, Cabre L, Ruiz J, Blanch L, Blanco J, Castillo F, et al. Indicadores de calidad en el paciente crítico. Med Intensiva. 2008;32:23-32.

15. Sánchez A, Palomar M, Alcaraz R, Moreira D. Infección nosocomial en unidad de cuidados intensivos " 0 ": iun objetivo alcanzable para todas las unidades? En: Net A y Quintana E, editores. Infecciones en Medicina Intensiva.
Barcelona: Ars Medica; 2007. p. 1-17. ISBN 978-84-9751274-9.

16. Insausti J, Palomar M, Álvarez F, Otal JJ, Olaechea P, López $M J$, et al. Estudio nacional de vigilancia de infección nosocomial en UCI. Informe del año 2008. Med Intensiva. 2009;33:109.

17. Fridkin SK, Hageman JC, Morrison M, Sanza LT, Como-Sabetti $\mathrm{K}$, Jeringan JA, et al. Methicillin-resistant Staphylococcus aureus disease in three communities. N Engl J Med. 2005;352: 1436-1444.

18. Cercenado E, Cuevas O, Marin M, Bouza E, Trincado P, Boquete $\mathrm{T}$, et al. Community-acquired methicillin-resistant Staphylococcus aureus in Madrid, Spain: Transcontinental importation and polyclonal emergence of Panton-Valentine leukocidin-positive isolates. Diagn Microbiol Infect Dis. 2008;61:143-9.

19. Oteo J, Cuevas O, Navarro C, Aracil B, Campos J. Trends in antimicrobial resistance in 3469 enterococci isolated from blood (EARSS experience 2001-06, Spain): Increasing ampicillin resistance in Enterococcus faecium. J Antimicrob Chemother. 2007;59:1044-5.

20. Maciá MD, Juan C, Oliver A, Hidalgo O, Pérez JL. Caracterización molecular de un brote por Enterococcus faecalis resistente a los glucopéptidos en una unidad de cuidados intensivos. Enferm Infecc Microbiol Clin. 2005;23:460-3.

21. Vincent JL, Brun-Buisson C, Niederman M, Haenni C, Harbarth $S$, Sprumont D, et al. Ethics roundtable debate: A patient dies from an ICU-acquired infection related to methicillin-resistant Staphylococcus aureus-how do you defend your case and your team? Crit Care. 2005;9:5-9.

22. Bryan CS, Call TJ, Elliott KC. The ethics of infection control: Philosophical frameworks. Infect Control Hosp Epidemiol. 2007;28:1077-84.

23. Haley RW, Schaberg DR, Crossley KB, Von Allmen SD, McGowan $\mathrm{Jr} \mathrm{JE}$. Extra charges and prolongation of stay attributable to nosocomial infections: A prospective interhospital comparison. Am J Med. 1981;70:51-8.

24. Wakefield DS, Pfaller M, Ludke RL, Wenzel RP. Methods for estimating days of hospitalization due to nosocomial infections. Med Care. 1992;30:373-6.

25. Delgado-Rodríguez $M$, Medina-Cuadros $M$, Bueno-Cavanillas A, Martínez-Gallego G, Dierssen T, Sillero-Arenas M. Comparison of two procedures to estimate the hospital stay attributable to nosocomial infection: Matched cohort study versus analysis of covariance of the total unmatched cohort. J Clin Epidemiol. 1997;50:773-8.

26. Blot S, Depuydt P, Vandewoude K, De Bacquer D. Measuring the impact of multidrug resistance in nosocomial infection. Curr Opin Infect Dis. 2007;20:391-6.

27. Fabbro-Peray $P$, Sotto A, Defez C, Cazaban M, Molinari L, Pinede $M$, et al. Mortality attributable to nosocomial infection: A cohort of patients with and without nosocomial infection in a French university hospital. Infect Control Hosp Epidemiol. 2007;28:265-72.

28. Blot S. Limiting the attributable mortality of nosocomial infection and multidrug resistance in intensive care units. Clin Microbiol Infect. 2008;14:5-13.

29. Garnacho-Montero J, Ortiz-Leyba C, Herrera-Melero I, AldaboPallas T, Cayuela-Domínguez A, Márquez-Vacaro JA, et al. Mortality and morbidity attributable to inadequate empirical antimicrobial therapy in patients admitted to the ICU with sepsis: A matched cohort study. J Antimicrob Chemother. 2008;61:436-41.

30. Sostarich AM, Zolldann D, Haefner H, Luetticken R, SchulzeRoebecke R, Lemmen SW. Impact of multiresistance of gramnegative bacteria in bloodstream infection on mortality rates and length of stay. Infection. 2008;36:31-5. 
31. Cosgrove SE. The relationship between antimicrobial resistance and patient outcomes: Mortality, length of hospital stay, and health care costs. Clin Infect Dis. 2006;42:S82-9.

32. McGregor JC, Rich SE, Harris AD, Perencevich EN, Osih R, Lodise Jr TP, et al. A systematic review of the methods used to assess the association between appropriate antibiotic therapy and mortality in bacteremic patients. Clin Infect Dis. 2007;45:329-37.

33. Ibrahim EH, Sherman G, Ward S, Fraser VJ, Kollef MH. The influence of inadequate antimicrobial treatment of bloodstream infections on patient outcomes in the ICU setting. Chest. 2000;118:146-55.

34. Harbarth S, Nobre V, Pittet D. Does antibiotic selection impact patient outcome? Clin Infect Dis. 2007;44:87-93.

35. Zaragoza R, Artero A, Camarena JJ, Sancho S, González R, Nogueira JM. The influence of inadequate empirical antimicrobial treatment on patients with bloodstream infections in an intensive care unit. Clin Microbiol Infect. 2003;9: $412-418$.

36. Osih RB, McGregor JC, Rich SE, Moore AC, Furuno J, Perencevich EN, et al. Impact of empiric antibiotic therapy on outcomes in patients with Pseudomonas aeruginosa bacteremia. Antimicrob Agents Chemother. 2007;51:839-44.

37. Fagon JY, Novara A, Stephan F, Girou E, Safar M. Mortality attributable to nosocomial infections in the ICU. Infect Control Hosp Epidemiol. 1994;15:428-34.

38. Salomao R, Rosenthal VD, Grimberg G, Coger S, Blecher S, Buchner-Ferreira $S$, et al. Device-associated infection rates in intensive care units of Brazilian hospitals: Findings of the International Nosocomial Infection Control Consortium. Rev Panam Salud Publica. 2008;24:195-202.

39. Cuellar LE, Fernández-Maldonado E, Rosenthal VD, CastanedaSabogal A, Rosales R, Mayorga-Espichan MJ, et al. Deviceassociated infection rates and mortality in intensive care units of Peruvian hospitals: Findings of the International Nosocomial Infection Control Consortium. Rev Panam Salud Publica. 2008;24:16-24.

40. Muscedere JG, Martin CM, Heyland DK. The impact of ventilator-associated pneumonia on the Canadian health care system. J Crit Care. 2008;23:5-10.

41. Valles J, Pobo A, García-Esquirol O, Mariscal D, Real J, Fernández R. Excess ICU mortality attributable to ventilatorassociated pneumonia: The role of early vs late onset. Intensive Care Med. 2007;33:1363-8.

42. Fagon JY, Chastre J, Hance AJ, Montravers P, Novara A, Gibert C. Nosocomial pneumonia in ventilated patients: A cohort study evaluating attributable mortality and hospital stay. Am J Med. 1993;94:281-8.

43. Rello J, Ollendorf DA, Oster G, Vera-Llonch M, Bellm L, Redman R, et al. Epidemiology and outcomes of ventilatorassociated pneumonia in a large US database. Chest. 2002;122:2115-21.

44. Moine P, Timsit JF, De Lassence A, Troche G, Fosse JP, Alberti $C$, et al. Mortality associated with late-onset pneumonia in the intensive care unit: Results of a multi-center cohort study. Intensive Care Med. 2002;28:154-63.

45. Leroy O, Meybeck A, D’Escrivan T, Devos P, Kipnis E, Georges $\mathrm{H}$. Impact of adequacy of initial antimicrobial therapy on the prognosis of patients with ventilator-associated pneumonia. Intensive Care Med. 2003;29:2170-3.

46. Álvarez-Lerma F. Modification of empiric antibiotic treatment in patients with pneumonia acquired in the intensive care unit. ICU-Acquired Pneumonia Study Group. Intensive Care Med. 1996;22:387-94.

47. Kollef MH, Silver P, Murphy DM, Trovillion E. The effect of lateonset ventilator-associated pneumonia in determining patient mortality. Chest. 1995;108:1655-62.
48. Kollef MH, Sherman G, Ward S, Fraser VJ. Inadequate antimicrobial treatment of infections: A risk factor for hospital mortality among critically ill patients. Chest. 1999;115:462-74.

49. Heyland DK, Cook DJ, Griffith L, Keenan SP, Brun-Buisson C. The attributable morbidity and mortality of ventilatorassociated pneumonia in the critically ill patient. The Canadian Critical Trials Group. Am J Respir Crit Care Med. 1999;159:1249-56.

50. Renaud B, Brun-Buisson C. Outcomes of primary and catheterrelated bacteremia. A cohort and case-control study in critically ill patients. Am J Respir Crit Care Med. 2001;163: 1584-1590.

51. Girou E, Stephan F, Novara A, Safar M, Fagon JY. Risk factors and outcome of nosocomial infections: Results of a matched case-control study of ICU patients. Am J Respir Crit Care Med. 1998;157:1151-8.

52. Pittet D, Tarara D, Wenzel RP. Nosocomial bloodstream infection in critically ill patients. Excess length of stay, extra costs, and attributable mortality. JAMA. 1994;271: 1598-601.

53. Di Giovine B, Chenoweth C, Watts C, Higgins M. The attributable mortality and costs of primary nosocomial bloodstream infections in the intensive care unit. Am J Respir Crit Care Med. 1999;160:976-81.

54. Rello J, Ochagavia A, Sabanes E, Roque M, Mariscal D, Reynaga $\mathrm{E}$, et al. Evaluation of outcome of intravenous catheterrelated infections in critically ill patients. Am J Respir Crit Care Med. 2000;162:1027-30.

55. Garrouste-Orgeas M, Timsit JF, Tafflet M, Misset B, Zahar JR, Soufir $L$, et al. Excess risk of death from intensive care unitacquired nosocomial bloodstream infections: A reappraisal. Clin Infect Dis. 2006;42:1118-26.

56. Olaechea PM, Álvarez-Lerma F, Palomar M, Otal JJ, Insausti J, López Pueyo MJ, et al. Mortalidad asociada a la bacteriemia primaria y relacionada con catéter. Estudio de cohortes emparejado. ENVIN-UCI 1997-2007. Med Intensiva. 2009; 33:109.

57. Bagshaw SM, Laupland KB. Epidemiology of intensive care unit-acquired urinary tract infections. Curr Opin Infect Dis. 2006;19:67-71.

58. Clec'h C, Schwebel C, Francais A, Toledano D, Fosse J P, Garrouste-Orgeas $M$, et al. Does catheter-associated urinary tract infection increase mortality in critically ill patients? Infect Control Hosp Epidemiol. 2007;28:1367-73.

59. Cosgrove SE, Sakoulas G, Perencevich EN, Schwaber MJ, Karchmer AW, Carmeli Y. Comparison of mortality associated with methicillin-resistant and methicillin-susceptible Staphylococcus aureus bacteremia: A meta-analysis. Clin Infect Dis. 2003;36:53-9.

60. Cosgrove SE, Qi Y, Kaye KS, Harbarth S, Karchmer AW, Carmeli Y. The impact of methicillin resistance in Staphylococcus aureus bacteremia on patient outcomes: Mortality, length of stay, and hospital charges. Infect Control Hosp Epidemiol. 2005;26:166-74.

61. Blot SI, Vandewoude KH, Hoste EA, Colardyn FA. Outcome and attributable mortality in critically ill patients with bacteremia involving methicillin-susceptible and methicillin-resistant Staphylococcus aureus. Arch Intern Med. 2002;162:2229-35.

62. Zahar JR, Clec'h C, Tafflet M, Garrouste-Orgeas M, Jamali S, Mourvillier $\mathrm{B}$, et al. Is methicillin resistance associated with a worse prognosis in Staphylococcus aureus ventilator-associated pneumonia? Clin Infect Dis. 2005;41:1224-31.

63. Soriano A, Martinez JA, Mensa J, Marco F, Almela M, MorenoMartínez A, et al. Pathogenic significance of methicillin resistance for patients with Staphylococcus aureus bacteremia. Clin Infect Dis. 2000;30:368-73. 
64. Combes A, Luyt CE, Fagon JY, Wolff M, Trouillet JL, Chastre J. Impact of methicillin resistance on outcome of Staphylococcus aureus ventilator-associated pneumonia. Am J Respir Crit Care Med. 2004;170:786-92.

65. Rello J, Sole-Violan J, Sa-Borges M, Garnacho-Montero J, Muñoz E, Sirgo G, et al. Pneumonia caused by oxacillinresistant Staphylococcus aureus treated with glycopeptides. Crit Care Med. 2005;33:1983-7.

66. Schramm GE, Johnson JA, Doherty JA, Micek ST, Kollef MH. Methicillin-resistant Staphylococcus aureus sterile-site infection: The importance of appropriate initial antimicrobial treatment. Crit Care Med. 2006;34:2069-74.

67. Lodise Jr TP, McKinnon PS. Burden of methicillin-resistant Staphylococcus aureus: Focus on clinical and economic outcomes. Pharmacotherapy. 2007;27:1001-12.

68. Shurland S, Zhan M, Bradham DD, Roghmann MC. Comparison of mortality risk associated with bacteremia due to methicillin-resistant and methicillin-susceptible Staphylococcus aureus. Infect Control Hosp Epidemiol. 2007;28:273-9.

69. Kang Cl, Kim SH, Park WB, Lee KD, Kim HB, Kim EC, et al. Risk factors for antimicrobial resistance and influence of resistance on mortality in patients with bloodstream infection caused by Pseudomonas aeruginosa. Microb Drug Resist. 2005;11:68-74.

70. Lautenbach E, Weiner MG, Nachamkin I, Bilker WB, Sheridan A, Fishman NO. Imipenem resistance among Pseudomonas aeruginosa isolates: Risk factors for infection and impact of resistance on clinical and economic outcomes. Infect Control Hosp Epidemiol. 2006;27:893-900.

71. Combes A, Luyt CE, Fagon JY, Wolff M, Trouillet JL, Chastre J. Impact of piperacillin resistance on the outcome of Pseudomonas ventilator-associated pneumonia. Intensive Care Med. 2006;32:1970-8.

72. Trouillet JL, Vuagnat A, Combes A, Kassis N, Chastre J, Gibert C. Pseudomonas aeruginosa ventilator-associated pneumonia: Comparison of episodes due to piperacillin-resistant versus piperacillin-susceptible organisms. Clin Infect Dis. 2002 1047-54.

73. Ibrahim EH, Sherman G, Ward S, Fraser VJ, Kollef MH. The influence of inadequate antimicrobial treatment of bloodstream infections on patient outcomes in the ICU setting. Chest. 2000;118:146-55.

74. Carmeli Y, Troillet N, Karchmer AW, Samore MH. Health and economic outcomes of antibiotic resistance in Pseudomonas aeruginosa. Arch Intern Med. 1999;159:1127-32.

75. Abbo A, Carmeli Y, Navon-Venezia S, Siegman-Igra Y, Schwaber MJ. Impact of multi-drug-resistant Acinetobacter baumannii on clinical outcomes. Eur J Clin Microbiol Infect Dis. 2007;26: 793-800.

76. Grupper M, Sprecher H, Mashiach T, Finkelstein R. Attributable mortality of nosocomial Acinetobacter bacteremia. Infect Control Hosp Epidemiol. 2007;28:293-8.

77. Playford EG, Craig JC, Iredell JR. Carbapenem-resistant Acinetobacter baumannii in intensive care unit patients: Risk factors for acquisition, infection and their consequences. $J$ Hosp Infect. 2007;65:204-11.

78. Lee NY, Lee HC, Ko NY, Chang CM, Shih HI, Wu CJ, et al. Clinical and economic impact of multidrug resistance in nosocomial Acinetobacter baumannii bacteremia. Infect Control Hosp Epidemiol. 2007;28:713-9.

79. Kollef KE, Schramm GE, Wills AR, Reichley RM, Micek ST, Kollef $\mathrm{MH}$. Predictors of 30-day mortality and hospital costs in patients with ventilator-associated pneumonia attributed to potentially antibiotic-resistant gram-negative bacteria. Chest. 2008;134:281-7.

80. Blot S, Vandewoude K, Colardyn F. Nosocomial bacteremia involving Acinetobacter baumannii in critically ill patients: A matched cohort study. Intensive Care Med. 2003;29:471-5.
81. Garnacho-Montero J, Ortiz-Leyba C, Fernández-Hinojosa E, Aldabo-Pallas T, Cayuela A, Márquez-Vacaro JA, et al. Acinetobacter baumannii ventilator-associated pneumonia: Epidemiological and clinical findings. Intensive Care Med. 2005;31:649-55.

82. Garnacho J, Sole-Violan J, Sa-Borges M, Díaz E, Rello J. Clinical impact of pneumonia caused by Acinetobacter baumannii in intubated patients: A matched cohort study. Crit Care Med. 2003;31:2478-82.

83. Melzer M, Petersen I. Mortality following bacteraemic infection caused by extended spectrum beta-lactamase (ESBL) producing $E$. coli compared to non-ESBL producing $E$. coli. J Infect. 2007;55:254-9.

84. Hyle EP, Lipworth AD, Zaoutis TE, Nachamkin I, Bilker WB, Lautenbach E. Impact of inadequate initial antimicrobial therapy on mortality in infections due to extended-spectrum beta-lactamase-producing enterobacteriaceae: Variability by site of infection. Arch Intern Med. 2005;165:1375-80.

85. Tumbarello M, Sali M, Trecarichi EM, Leone F, Rossi M, Fiori B, et al. Bloodstream infections caused by extended-spectrum beta-lactamase-producing Escherichia coli: Risk factors for inadequate initial antimicrobial therapy. Antimicrob Agents Chemother. 2008;52:3244-52.

86. Kang $\mathrm{Cl}$, Kim SH, Park WB, Lee KD, Kim HB, Kim EC, et al. Bloodstream infections due to extended-spectrum betalactamase-producing Escherichia treatment outcome, with special emphasis on antimicrobial therapy. Antimicrob Agents Chemother. 2004;48:4574-81.

87. Pena C, Gudiol C, Calatayud L, Tubau F, Domínguez MA, Pujol $M$, et al. Infections due to Escherichia coli producing extended-spectrum beta-lactamase among hospitalised patients: Factors influencing mortality. J Hosp Infect. 2008 116-22.

88. Kola A, Maciejewski O, Sohr D, Ziesing S, Gastmeier P. Clinical impact of infections caused by ESBL-producing $\mathrm{E}$. coli and $\mathrm{K}$. pneumoniae. Scand J Infect Dis. 2007;39:975-82.

89. Schwaber MJ, Carmeli Y. Mortality and delay in effective therapy associated with extended-spectrum beta-lactamase production in Enterobacteriaceae bacteraemia: A systematic review and meta-analysis. J Antimicrob Chemother. 2007 913-20.

90. Olaechea PM, Ulibarrena MA, Álvarez-Lerma F, Insausti J, Palomar M, De la Cal MA. Factors related to hospital stay among patients with nosocomial infection acquired in the intensive care unit. Infect Control Hosp Epidemiol. 2003 207-13.

91. DiGiovine B, Chenoweth C, Watts C, Higgins M. The attributable mortality and costs of primary nosocomial bloodstream infections in the intensive care unit. Am J Respir Crit Care Med. 1999;160:976-81.

92. Pittet D, Tarara D, Wenzel RP. Nosocomial bloodstream infection in critically ill patients. Excess length of stay, extra costs, and attributable mortality. JAMA. 1994;271:1598-601.

93. Kirkland KB, Weinstein JM. Adverse effects of contact isolation. Lancet. 1999;354:1177-8.

94. Stelfox HT, Bates DW, Redelmeier DA. Safety of patients isolated for infection control. JAMA. 2003;290:1899-905.

95. Olaechea PM, Palomar M, León-Gil C, Álvarez-Lerma F, Jorda $\mathrm{R}$, Nolla-Salas $\mathrm{J}$, et al. Economic impact of Candida colonization and Candida infection in the critically ill patient. Eur J Clin Microbiol Infect Dis. 2004;23:323-30.

96. Bantar C, Sartori B, Vesco E, Heft C, Saul M, Salamote F, et al. A hospitalwide intervention program to optimize the quality of antibiotic use: Impact on prescribing practice, antibiotic consumption, cost savings, and bacterial resistance. Clin Infect Dis. 2003;37:180-6. 
97. Cosgrove SE. The relationship between antimicrobial resistance and patient outcomes: Mortality, length of hospital stay, and health care costs. Clin Infect Dis. 2006;42:S82-9.

98. Kilgore ML, Ghosh K, Beavers CM, Wong DY, Hymel Jr PA, Brossette SE. The costs of nosocomial infections. Med Care. 2008;46:101-4.

99. Sánchez-Velázquez LD, Ponce de León RS, Rangel Frausto MS. The burden of nosocomial infection in the intensive care unit: Effects on organ failure, mortality and costs. A nested casecontrol study. Arch Med Res. 2006;37:370-5.
100. Lee SY, Kotapati S, Kuti JL, Nightingale CH, Nicolau DP. Impact of extended-spectrum beta-lactamase-producing Escherichia coli and Klebsiella species on clinical outcomes and hospital costs: A matched cohort study. Infect Control Hosp Epidemiol. 2006;27:1226-32.

101. Jain M, Miller L, Belt D, King D, Berwick DM. Decline in ICU adverse events, nosocomial infections and cost through a quality improvement initiative focusing on teamwork and culture change. Qual Saf Health Care. 2006;15: 235-9. 